\title{
Association of healthy eating index with metabolic syndrome and its components among type 2 diabetes patients in Gaza Strip, Palestine: A cross sectional study
}

\author{
Abdel Hamid El Bilbeisi ${ }^{1,2 *}$, Amany El Afifi ${ }^{3}$ and Kurosh Djafarian ${ }^{2}$ \\ ${ }^{1}$ Department of Clinical Nutrition, Faculty of Pharmacy, Al Azhar University of Gaza, Palestine \\ ${ }^{2}$ Department of Clinical Nutrition, School of Nutritional Sciences and Dietetics, Tehran University of Medical Sciences, International Campus (TUMS- IC), \\ Tehran, Iran \\ ${ }^{3}$ Faculty of Pharmacy, Al Azhar University of Gaza, Palestine
}

\begin{abstract}
The prevalence of metabolic syndrome is raising worldwide; however, the role of diet in the origin of metabolic syndrome is not understood well. This study was conducted to determine the association between Healthy Eating Index-2010 (HEI-2010) with metabolic syndrome and its components among type 2 diabetes patients in Gaza Strip, Palestine. This cross sectional study was conducted among 1187 previously diagnosed type 2 diabetes mellitus (both genders, aged 20-64 years), patients receiving care in primary healthcare centers in Gaza Strip, Palestine. A validated semi-quantitative food frequency questionnaire was used to assess the dietary intakes of patients. HEI-2010 was used to assess diet quality of patients. Metabolic syndrome was defined based on the International Diabetes Federation criteria. Additional information regarding demographic and medical history variables was obtained with an interview-based questionnaire. Statistical analysis was performed using SPSS version 20. After adjustment for potential confounders, patients in the lowest quartile (Q1) of HEI-2010 had the highest risk of (Metabolic syndrome, central obesity, high triglycerides, low HDL-cholesterol and high blood pressure), (Odds ratio $[\mathrm{OR}]=1.804,95 \%$ confidence interval $[\mathrm{CI}]=[1.494-2.178]),(\mathrm{OR}=$ $1.351,95 \% \mathrm{CI}=[1.077-1.694]),(\mathrm{OR}=2.270,95 \% \mathrm{CI}=[1.911-2.697]),(\mathrm{OR}=2.089,95 \% \mathrm{CI}=[1.763-2.475]),(\mathrm{OR}=1.361,95 \% \mathrm{CI}=[1.145-1.618])$, respectively, ( $\mathrm{P}$ value $<0.005$ for all), compared with those in the upper quartile (Q4). In conclusion, the highest HEI-2010 score may be associated with a lower prevalence of metabolic syndrome and its components among type 2 diabetes patients.
\end{abstract}

\begin{abstract}
Abbreviations: HEI-2010: Healthy eating index-2010; Q: Quartile; OR: Odds ratio; CI: Confidence interval; Mets: Metabolic syndrome; IDF: International diabetes federation; T2DM: Type 2 diabetes mellitus; PHCs: Primary healthcare centers; FFQ: Food frequency questionnaire; WC: Waist circumference; BMI: Body mass index; BP: Blood pressure; IPAQ: International physical activity questionnaire; MET: Metabolic equivalents; FPG: Fasting plasma glucose; HDL-c: High-density lipoprotein cholesterol; TGs: Triglycerides.
\end{abstract}

\section{Introduction}

Metabolic syndrome (MetS) is a collection of cardiovascular risk factors, which is related to increased risk of diabetes, stroke and mortality [1]. MetS has shown an increasing prevalence globally because of the constant increase of health-related factors such as obesity and physical inactivity $[1,2]$. MetS is a major health problem worldwide; based on the International Diabetes Federation (IDF) appreciation about one quarter of the world's adult population have MetS [3]. Several studies show that, the prevalence of MetS among type 2 diabetes mellitus (T2DM) patients is more than double of the prevalence in the general populations [4,5]. MetS is a multifactorial disorder; several factors including genetic, metabolic and environmental factors can contribute to the etiology of MetS [1]. Therefore, finding modifiable risk factors of MetS is of great importance. Among the modifiable risk factors, dietary intakes have been highlighted as an effective approach for prevention and management of MetS [6]. Previous studies have widely reviewed the effect of several single foods, and nutrients on MetS and its components [7-9]. These studies provide beneficial data about the effects of dietary intake on MetS development but the obtained results may not be applicable for MetS management. As single foods and nutrients are not usually consumed alone, studying the dietary patterns provides opportunity to consider the effects of whole diet on MetS and its components and offer a functional approach for MetS management [6]. Dietary pattern is potentially useful in making dietary recommendations because overall dietary patterns might be easy for the public to interpret or translate into diets [10].

On the other hand, an impressive body of evidence from epidemiological and clinical trials indicated that consumption of foods rich in antioxidants, omega-3, or minerals rather than sodium, present in the Mediterranean diet pattern protect people against MetS and its components $[11,12]$. The cumulative results of these studies highlight the importance of diet quality in reducing risk factors for cardiometabolic disorders [11,12]. Diet quality indices are widely used to evaluate the relationship between total diet and chronic diseases risk factors [13].

*Correspondence to: Abdel Hamid el Bilbeisi, Department of Clinical Nutrition, Faculty of Pharmacy, Al Azhar University of Gaza, Palestine, Tel: 0098(0)21 88955975; Fax: 0098(0)2188955975; E-mail: Abed_az@hotmail.com

Key words: diabetes mellitus, healthy eating index, metabolic syndrome, Palestine Received: October 14, 2019; Accepted: November 01, 2019; Published: November 04, 2019 
In addition, the majority of studies used the Healthy Eating Index-2010 (HEI-2010) for evaluation of diet quality $[13,14]$. The HEI-2010 is an updated tool for assessing diet quality as specified by the Dietary Guidelines for Americans, that contains twelve main components and a total score of one hundred points [15]. The HEI-2010 can be used for a variety of applications, including population monitoring [16]; epidemiologic research [17]; and evaluations of the food environment [18], food assistance packages, nutrition interventions [19], and the relation between diet cost and diet quality [20]. Furthermore, the HEI2010 is a summary measure of diet quality; it's a simple facility for monitoring the changes in eating habits and decision-making in order to improve nutrition [21,22]. Schwingshackl et al. [23] in the updated meta-analyses show that, diets that score highly on the HEI-2010 were associated with a significant reduction in the risk of all-cause mortality, cardiovascular disease, cancer, T2DM, and neurodegenerative disease by $22 \%, 22 \%, 16 \%, 18 \%$, and $15 \%$, respectively. Furthermore, highquality diets were inversely associated with overall mortality and cancer mortality among cancer survivors. Additionally, Onvani et al. [24] in a meta-analysis show that, the highest level of adherence to the HEI-2010 was significantly associated with a reduced risk of all-cause mortality, cardiovascular mortality and cancer mortality.

In Palestine, El Bilbeisi et al. [25] in a meta-analysis show that, MetS is highly prevalent $(37.0 \%)$ among Palestinian adults. However, dietary intakes of Middle-Eastern population have their own characteristics: Large intake of carbohydrates mostly in the form of refined grains, high intakes of trans and saturated fat, and low consumption of fruits and vegetables along with lack of alcohol intake makes their dietary pattern of great interest for the assessment of diet-disease relations [26]. Moreover, different from Western people, stressful life is highly prevalent in this region, which might further complicate diet-disease relations in this area [27]. Therefore, understanding the association between HEI-2010 with MetS and its component may be helpful in reducing disease-related premature mortality and improve outcomes among T2DM patients. To the best of our knowledge, there is no data about the association of HEI-2010 score with MetS and its components among T2DM patients in Gaza Strip, Palestine. This study was conducted to determine the association between HEI-2010 with MetS and its components among T2DM patients.

\section{Materials and methods}

\section{Study design and study population}

This cross sectional study was conducted in the years 2015 and 2016 among a representative sample of Palestinian T2DM patients, selected by a cluster random sampling method. A total of 1187 patients, aged 20-64 years receiving care in the primary healthcare centers (PHCs) in Gaza Strip, Palestine, were included in the study. Gaza Strip is divided into five smaller governorates, which include North Gaza, Gaza City, Mid Zone, Khan Younis and Rafah. The total number of PHCs in Gaza Strip is fifty-four [3]. The PHCs were distributed in each governorate as follows (Eight, fourteen, sixteen, eleven and five PHCs respectively). The study sample was distributed according to the number of PHCs in each governorate as follows (176, 308, 353, 241 and 109 patients respectively). Pregnant, lactating women, patients with total energy intake $<800$ and $>4200 \mathrm{kcal}$, and patients with other types of serious illness such as cancer or acute myocardial infarction were excluded from the study.

\section{Assessment of dietary intake}

Data about dietary intake were collected by an expert nutritionist, using a validated semi-quantitative food frequency questionnaire
(FFQ). The FFQ in our study contains a list of 98 food items; it was developed and validated among Palestinian population in 2014 [10]. All participants were asked to estimate the number of times per day, week or month he/she consumed these particular food products and the amount usually eaten per food item by making comparisons with the specified reference portion. Common household measures including measuring cups, spoons and a ruler were shown to assist the participants in the estimation process. The answer categories ranged from 1 to 7 times ( 7 categories) including never, one to three times per month, one to two times per week, three to four times per week, five to six times per week, one time per day or two to three times per day. The food composition of mixed dishes was determined by using common recipes consumed in the country. The mean intake of each food item in grams was calculated by multiplying specified portion size by the average reported frequency.

\section{Healthy eating index-2010 (HEI-2010)}

The HEI-2010 is an updated tool for assessing diet quality as specified by the 2010 Dietary Guidelines for Americans, that contains 12 main components and a total score of 100 points [15]. Total fruits, whole fruits, total vegetables, greens and beans, total protein foods and seafood and plant proteins scored 5 in the highest consumption and 0 in the lowest consumption. The highest consumption of three components including whole grains, dairy and fatty acid scored as 10 and the lowest consumption of them scored as 0 . Two components (Refined grains and sodium) scored 10 in the lowest consumption and 0 in the highest consumption. One component that is, empty calories (Energy from solid fats, added sugars and any alcohol in excess of 13 $\mathrm{g} / 1000 \mathrm{kcal}$ ) scored $0-20$. The highest frequency of consumption scored as 0 and the lowest frequency of consumption scored as 20 . The total HEI-2010 score was calculated by adding the 12 components' score [28]. The USDA food composition tables were used to analyze nutrients consumption [29]. For all components, higher scores reflect better diet quality and greater adherence to Dietary Guidelines for Americans recommendations [15].

\section{Assessment of anthropometric measurements}

Height was measured in all patients (Patients bare footed and head upright) with a measuring rod attached to the balanced beam scale; the height was reported to the nearest $0.5 \mathrm{~cm}$ [30]. Weight $(\mathrm{kg})$ was measured using standard scale (Seca); the scale was placed on a hardfloor surface; patients were asked to remove their heavy outer garments and weight was measured and recorded to the nearest $0.1 \mathrm{~kg}$ [31]. Furthermore, a stretch-resistant tape was used for measuring waist circumference (WC); WC was measured at the approximate midpoint between the lower margin of the last palpable rib and the top of the iliac crest [32]. The body mass index (BMI) was calculated by dividing weight in kilograms by the square of height in meters [32].

\section{Assessment of blood pressure (BP)}

The systolic and diastolic BP was measured from the left arm (mmHg) by mercury sphygmomanometer [33]. Three readings on different days, while the patient was seated after relaxing for at least fifteen minutes in a quiet environment, empty bladder. The average of the measurements was recorded.

\section{Assessment of physical activity}

Data on physical activity were obtained using the International Physical Activity Questionnaire (IPAQ short version) [34]. The internationally accepted protocol was used to estimate the weekly 
calorie expenditure expressed as metabolic equivalents per week (MET/ wk). The IPAQ scoring protocol assigns the following MET values to walking, moderate and vigorous intensity activity: 3.3 METs, 4.0 METs, and 8.0 METs, respectively [34].

\section{Biochemical analysis}

After 12 hours fasting, venous blood samples were collected from all patients in the PHCs (In the second meeting with the patients) by well-trained and experienced nurses. Venous blood $(4.0 \mathrm{ml})$ was drawn into vacationer tubes and was used for blood chemistry analysis. Serum was separated immediately, and the extracted serum was investigated for (Fasting Plasma Glucose (FPG) mg/dl, High-Density Lipoprotein Cholesterol (HDL-c) mg/dl and Triglycerides (TGs) mg/dl). Mindray BS-300 chemistry analyzer instrument was used for blood chemistry analysis [3]. The laboratory tests were analyzed in a private licensed laboratory.

\section{Assessment of other variables}

Additional information regarding demographic and medical history variables was obtained with an interview-based questionnaire. Moreover, reports and all relevant documentation, including medical records were checked. Pilot study was carried out on thirty patients to enable the researcher to examine the tools of the study. The questionnaire and data collection process were modified according to the result of the pilot study. The data was collected by ten qualified data collectors (Five nurses and five nutritionists), who were given a full explanation and training by the researcher about the study.

\section{Definition of metabolic syndrome (MetS)}

MetS was defined based on the IDF criteria [35]. However, in this study all participants were previously diagnosed patients with T2DM under management and therefore, were classified as hyperglycemic irrespective of the level of FPG at the time of sampling.

\section{Statistical analysis}

Statistical analysis was performed using SPSS version 20. Normal distribution of data was checked by histogram curves and KolmogorovSmirnov test. In this study, all variables had normal distribution. Patients were categorized based on quartile cut-points of HEI-2010 including $\leq 63,64-67.5,67.51-73$ and $\geq 74$ [36]. Characteristics of the study population across quartiles of HEI-2010 were examined using analysis of variance for continuous variables or chi-square test for categorical variables. Age and energy adjusted food and nutrients consumption across quartiles of HEI-2010 were calculated using analysis of covariance (One-way ANCOVA). Finally, the odds ratio (OR) and 95\% confidence interval (CI) for the MetS and its components across quartiles of HEI-2010 were tested by binary logistic regression. P value less than 0.05 was considered as statistically significant.

\section{Results}

A total of 1187 patients with T2DM aged 20 to 64 years old (59.7\% females, $40.3 \%$ males) were included in this study. The characteristics of study population across HEI-2010 quartiles are shown in Table 1. The results revealed that the mean age (years) for patients in the lowest quartile (Q1) was $54.0 \pm 9.1$ vs. $43.8 \pm 10.9$ for patients in the upper

Table 1. Characteristics of study population by quartiles of HEI-2010

\begin{tabular}{|c|c|c|c|c|c|c|}
\hline \multirow{2}{*}{\multicolumn{2}{|c|}{ Variables }} & \multicolumn{4}{|c|}{ Quartiles of Healthy Eating Index-2010 } & \multirow{3}{*}{$\begin{array}{r}\text { P value } \\
0.001 \\
\end{array}$} \\
\hline & & \multirow{2}{*}{$\begin{array}{c}\text { Q1 (Lowest) } \\
\text { No. (297) } \\
54.0 \pm 9.1\end{array}$} & \multirow{2}{*}{$\begin{array}{c}\text { Q2 } \\
\text { No. (299) } \\
51.4 \pm 10.0\end{array}$} & \multirow{2}{*}{$\begin{array}{c}\text { Q3 } \\
\text { No. (296) } \\
49.8 \pm 11.4\end{array}$} & \multirow{2}{*}{$\begin{array}{c}\text { Q4 (Highest) } \\
\text { No. (295) } \\
43.8 \pm 10.9\end{array}$} & \\
\hline Age (years) & Mean \pm SD & & & & & \\
\hline \multirow{2}{*}{ Gender } & Male & $103.0(21.6)$ & $114.0(23.8)$ & $121.0(25.3)$ & $140.0(29.3)$ & \multirow[b]{2}{*}{0.013} \\
\hline & Female & $194.0(27.4)$ & $185.0(26.1)$ & $175.0(24.7)$ & $155.0(21.8)$ & \\
\hline \multirow{2}{*}{ Marital status } & Married & $289.0(25.2)$ & $291.0(25.4)$ & $285.0(24.8)$ & $282.0(24.6)$ & \multirow[b]{2}{*}{0.584} \\
\hline & Unmarried & $8.0(20.0)$ & $8.0(20.0)$ & $11.0(27.5)$ & $13.0(32.5)$ & \\
\hline \multirow{2}{*}{ Educational level } & Low education & $150.0(28.3)$ & $159.0(30.0)$ & $127.0(24.0)$ & $94.0(17.7)$ & \multirow[b]{2}{*}{0.001} \\
\hline & High education & $147.0(22.4)$ & $140.0(21.3)$ & $169.0(25.7)$ & $201.0(30.6)$ & \\
\hline \multirow{2}{*}{ Family size } & Less than five & $94.0(22.1)$ & $103.0(24.2)$ & $105.0(24.6)$ & $124.0(29.1)$ & \multirow[b]{2}{*}{0.059} \\
\hline & Five or more & $203.0(26.7)$ & $196.0(25.7)$ & $191.0(25.1)$ & $171.0(22.5)$ & \\
\hline \multirow{2}{*}{ Monthly income } & $\leq 2000(\mathrm{NIS})$ & $272.0(26.0)$ & $262.0(25.1)$ & $270.0(25.9)$ & $240.0(23.0)$ & \multirow[b]{2}{*}{0.001} \\
\hline & $>2000$ (NIS) & $25.0(17.5)$ & $37.0(25.9)$ & $26.0(18.1)$ & $55.0(38.5)$ & \\
\hline \multirow{3}{*}{ Diabetes duration (years) } & Less than five & $52.0(16.7)$ & $58.0(18.6)$ & $69.0(22.2)$ & $132.0(42.5)$ & \multirow{3}{*}{0.001} \\
\hline & Five to ten & $88.0(20.0)$ & $111.0(25.1)$ & $111.0(25.1)$ & $132.0(29.8)$ & \\
\hline & More than ten & $157.0(35.3)$ & $130.0(29.2)$ & $116.0(26.1)$ & $42.0(9.4)$ & \\
\hline Use diabetes medications & Yes & $297.0(25.0)$ & $299.0(25.2)$ & $296.0(24.9)$ & $295.0(24.9)$ & - \\
\hline \multirow{3}{*}{ Type of diabetes medications used } & Diabetes pills & $117.0(24.1)$ & $106.0(21.8)$ & $109.0(22.4)$ & $154.0(31.7)$ & \multirow{3}{*}{0.001} \\
\hline & Insulin injections & $156.0(25.1)$ & $171.0(27.5)$ & $162.0(26.0)$ & $133.0(21.4)$ & \\
\hline & Pills \& injections & $24.0(30.4)$ & $22.0(27.8)$ & $25.0(31.7)$ & $8.0(10.1)$ & \\
\hline \multirow{2}{*}{ Received diabetes care instructions } & Yes & $121.0(21.5)$ & $139.0(24.7)$ & $148.0(26.3)$ & $155.0(27.5)$ & \multirow[b]{2}{*}{0.025} \\
\hline & No & $176.0(28.3)$ & $160.0(25.6)$ & $148.0(23.7)$ & $140.0(22.4)$ & \\
\hline \multirow{2}{*}{ History of smoking } & Yes & $34.0(21.1)$ & $42.0(26.1)$ & $38.0(23.6)$ & $47.0(29.2)$ & \multirow[b]{2}{*}{0.434} \\
\hline & No & $263.0(25.6)$ & $257.0(25.0)$ & $258.0(25.2)$ & $248.0(24.2)$ & \\
\hline History of alcohol intake & No & $0.0(0.0)$ & $0.0(0.0)$ & $0.0(0.0)$ & $0.0(0.0)$ & - \\
\hline Physical activity (total MET) & Mean \pm SD & $1049.5 \pm 1328$ & $1024.8 \pm 1326$ & $1568.8 \pm 1669$ & $2074.7 \pm 1483$ & 0.001 \\
\hline Body mass index $\left(\mathrm{kg} / \mathrm{m}^{2}\right)$ & Mean \pm SD & $31.7 \pm 6.15$ & $31.7 \pm 6.12$ & $30.0 \pm 6.48$ & $27.2 \pm 5.11$ & 0.002 \\
\hline FPG $(\mathrm{mg} / \mathrm{dl})$ & Mean \pm SD & $173.4 \pm 30$ & $167.6 \pm 27$ & $171.5 \pm 31$ & $166.1 \pm 26$ & 0.006 \\
\hline
\end{tabular}

Data are expressed as means \pm SD for continuous variables and as percentage for categorical variables. The characteristics of the study population across quartiles of HEI-2010 were examined using analysis of variance for continuous variables or chi-square test for categorical variables. P value less than 0.05 was considered as statistically significant. Q, quartile; SD, stander deviation; MET, metabolic equivalent; FPG, fasting plasma glucose. 
El Bilbeisi AH (2019) Association of healthy eating index with metabolic syndrome and its components among type 2 diabetes patients in Gaza Strip, Palestine: A cross sectional study

quartile (Q4). In comparison with the upper quartile, those who were assigned to the lowest quartile of HEI-2010 were older, had lower educational level, had lower monthly income, had higher diabetes duration, had lower rate of physical activity, had higher FPG level, and were more likely to be obese. In addition, for the following factors (Age, gender, educational level, monthly income, diabetes duration, type of diabetes medications used, received diabetes care instructions, physical activity, BMI and FPG), the difference was statistically significant a cross quartiles of HEI-2010 (P value $<0.05$ for all). On the other hand, the distribution of study population by MetS and its components across quartiles of HEI-2010 are shown in Table 2. Our results revealed that, $62.5 \%$ of T2DM patients had MetS based on the IDF criteria. Furthermore, in comparison with the upper quartile, patients who were assigned to the lowest quartile of HEI-2010 had higher prevalence of MetS (33.0 vs. 12.3\%), central obesity (28.4 vs. 20.0\%), high TGs levels (37.3 vs. $0.8 \%$ ), low HDL-c levels (36.3 vs. 0.4 ), and had higher BP or treatment of previously diagnosed hypertension (30.7 vs. 13.9\%). Moreover, for the following factors (MetS, central obesity, high TGs, low HDL-c and high BP or treatment of previously diagnosed hypertension), the difference was statistically significant a cross quartiles of HEI-2010 (P value $<0.05$ for all).
Additionally, the dietary intakes of study population across quartiles of HEI-2010 are presented in Table 3. Our findings revealed that, patients in the upper quartile of HEI-2010 had higher intakes of carbohydrate ( $237 \pm 27$ vs. $219 \pm 17)$, dietary fiber $(21 \pm 4$ vs. $8 \pm 2)$, fruits ( $244 \pm 81$ vs. $147 \pm 34)$, vegetables ( $292 \pm 69$ vs. $189 \pm 73)$, meat and fish ( $152 \pm 78$ vs. $124 \pm 57)$, whole grains $(84 \pm 27$ vs. $30 \pm 12)$, and nuts \& legumes ( $85 \pm 16$ vs. $35 \pm 12)$, compared with those in the lowest quartile (P value $<0.05$ for all). On the other hand, Table 3 show that, the energy intake, fat and refined grain consumption decreased significantly across HEI-2010 quartiles (P value $<0.05$ for all). No significant association was found between HEI-2010 quartiles with protein, cholesterol and dairy consumption. Finally, we computed the OR and 95\% CI for the MetS and its components across quartiles of HEI-2010 (Table 4). Our findings demonstrate that, after adjustment for potential confounders, patients in the lowest quartile of HEI-2010 had the highest risk of (MetS, central obesity, high TGs levels, low HDL-c levels and high $\mathrm{BP}$ or treatment of previously diagnosed hypertension), $(\mathrm{OR}=1.804$, $95 \% \mathrm{CI}=[1.494-2.178]),(\mathrm{OR}=1.351,95 \% \mathrm{CI}=[1.077-1.694]),(\mathrm{OR}=$ $2.270,95 \% \mathrm{CI}=[1.911-2.697]),(\mathrm{OR}=2.089,95 \% \mathrm{CI}=[1.763-2.475])$, $(\mathrm{OR}=1.361,95 \% \mathrm{CI}=[1.145-1.618])$, respectively, $(\mathrm{P}$ value $<0.005$ for all), compared with those in the upper quartile.

Table 2. Distribution of study population by metabolic syndrome and its components across quartiles of HEI-2010

\begin{tabular}{|c|c|c|c|c|c|c|}
\hline \multirow{2}{*}{\multicolumn{2}{|c|}{ Variables }} & \multicolumn{4}{|c|}{ Quartiles of Healthy Eating Index-2010 } & \multirow[b]{2}{*}{ P value } \\
\hline & & \multirow{2}{*}{$\begin{array}{c}\text { Q1 (Lowest) } \\
\text { No. (297) } \\
245.0(33.0)\end{array}$} & \multirow{2}{*}{$\begin{array}{c}\text { Q2 } \\
\text { No. (299) } \\
229.0(30.9)\end{array}$} & \multirow{2}{*}{$\begin{array}{c}\text { Q3 } \\
\text { No. (296) } \\
177.0(23.8)\end{array}$} & \multirow{2}{*}{$\begin{array}{c}\text { Q4 (Highest) } \\
\text { No. (295) } \\
91.0(12.3)\end{array}$} & \\
\hline \multirow{2}{*}{ Metabolic syndrome } & Yes & & & & & \multirow[b]{2}{*}{0.001} \\
\hline & No & $52.0(11.7)$ & $70.0(15.8)$ & $119.0(26.7)$ & $204.0(45.8)$ & \\
\hline \multirow{2}{*}{ Central obesity (waist circumference $>90 \mathrm{~cm}$ for men or $>80 \mathrm{~cm}$ for women) } & Yes & $277.0(28.4)$ & $262.0(26.8)$ & $242.0(24.8)$ & $195.0(20.0)$ & \multirow[b]{2}{*}{0.001} \\
\hline & No & $20.0(9.5)$ & $37.0(17.5)$ & $54.0(25.6)$ & $100.0(47.4)$ & \\
\hline \multirow{2}{*}{ Triglycerides $\geq 150 \mathrm{mg} / \mathrm{dl}$ or specific treatment for this lipid abnormality } & Yes & $186.0(37.3)$ & $175.0(35.1)$ & $134.0(26.8)$ & $4.0(0.8)$ & \multirow[b]{2}{*}{0.001} \\
\hline & No & $111.0(16.1)$ & $124.0(18.0)$ & $162.0(23.6)$ & $291.0(42.3)$ & \\
\hline \multirow{2}{*}{$\begin{array}{l}\mathrm{HDL}-\mathrm{c}<40 \mathrm{mg} / \mathrm{dl} \text { in males and }<50 \mathrm{mg} / \mathrm{dl} \text { in females or specific treatment } \\
\text { for this lipid abnormality }\end{array}$} & Yes & $175.0(36.3)$ & $173.0(35.9)$ & $132.0(27.4)$ & $2.0(0.4)$ & \multirow[b]{2}{*}{0.001} \\
\hline & No & $122.0(17.3)$ & $126.0(17.9)$ & $164.0(23.3)$ & $293.0(41.5)$ & \\
\hline \multirow{2}{*}{$\begin{array}{l}\text { High blood pressure }(\geq 130 / 85 \mathrm{mmHg} \text { ) or treatment of previously diagnosed } \\
\text { HTN }\end{array}$} & Yes & $235.0(30.7)$ & $238.0(31.1)$ & $186.0(24.3)$ & $106.0(13.9)$ & \multirow{3}{*}{0.001} \\
\hline & No & $62.0(14.7)$ & $61.0(14.4)$ & $110.0(26.1)$ & $189.0(44.8)$ & \\
\hline \multicolumn{6}{|c|}{ In our study, all participants previously diagnosed T2DM } & \\
\hline
\end{tabular}

Data are expressed as percentage for categorical variables. The chi-square test was used to examine differences in the prevalence of different categorical variable. $\mathrm{P}$ value less than 0.05 was considered as statistically significant. Q, quartile; HDL-c, high-density lipoprotein cholesterol; HTN, hypertension.

Table 3. Dietary intakes of study population by quartiles of HEI-2010

\begin{tabular}{|c|c|c|c|c|c|c|}
\hline \multirow{2}{*}{\multicolumn{2}{|c|}{ Food and nutrients }} & \multicolumn{4}{|c|}{ Quartiles of Healthy Eating Index-2010 } & \multirow{3}{*}{$\begin{array}{r}\text { P value } \\
0.001 \\
\end{array}$} \\
\hline & & \multirow{2}{*}{$\begin{array}{c}\text { Q1 (Lowest) } \\
\text { No. (297) } \\
2538 \pm 303 \\
\end{array}$} & \multirow{2}{*}{$\begin{array}{c}\text { Q2 } \\
\text { No. (299) } \\
2360 \pm 141\end{array}$} & \multirow{2}{*}{$\begin{array}{c}\text { Q3 } \\
\text { No. (296) } \\
2274 \pm 212 \\
\end{array}$} & \multirow{2}{*}{$\begin{array}{c}\text { Q4 (Highest) } \\
\text { No. (295) } \\
2133 \pm 198 \\
\end{array}$} & \\
\hline Total energy (kcal per day) & Mean \pm SD & & & & & \\
\hline Carbohydrate (g per day) & Mean \pm SD & $219 \pm 17$ & $227 \pm 20$ & $232 \pm 24$ & $237 \pm 27$ & 0.001 \\
\hline Protein (g per day) & Mean \pm SD & $72 \pm 9$ & $73 \pm 12$ & $76 \pm 17$ & $73 \pm 12$ & 0.160 \\
\hline Fat (g per day) & Mean \pm SD & $118 \pm 32$ & $103 \pm 28$ & $86 \pm 35$ & $82 \pm 37$ & 0.001 \\
\hline Cholesterol (mg per day) & Mean \pm SD & $252 \pm 58$ & $255 \pm 62$ & $254 \pm 73$ & $246 \pm 60$ & 0.210 \\
\hline Dietary fiber (g per day) & Mean \pm SD & $8 \pm 2$ & $13 \pm 3$ & $17 \pm 4$ & $21 \pm 4$ & 0.001 \\
\hline Fruits & Mean \pm SD & $147 \pm 34$ & $183 \pm 56$ & $233 \pm 78$ & $244 \pm 81$ & 0.001 \\
\hline Vegetables & Mean \pm SD & $189 \pm 73$ & $264 \pm 95$ & $267 \pm 91$ & $292 \pm 69$ & 0.001 \\
\hline Meat and fish & Mean \pm SD & $124 \pm 57$ & $142 \pm 68$ & $145 \pm 74$ & $152 \pm 78$ & 0.001 \\
\hline Whole grains & Mean \pm SD & $30 \pm 12$ & $42 \pm 22$ & $75 \pm 19$ & $84 \pm 27 \uparrow$ & 0.001 \\
\hline Refined grains & Mean \pm SD & $395 \pm 72$ & $360 \pm 92$ & $337 \pm 129$ & $332 \pm 138$ & 0.001 \\
\hline Dairy & Mean \pm SD & $239 \pm 89$ & $269 \pm 68$ & $281 \pm 85$ & $251 \pm 78$ & 0.080 \\
\hline Nut and legumes & Mean \pm SD & $35 \pm 12$ & $51 \pm 21$ & $64 \pm 25$ & $85 \pm 16$ & 0.001 \\
\hline
\end{tabular}

Food and nutrients consumption across quartiles of HEI-2010 were calculated using analysis of covariance (One-way ANCOVA). *Adjusted for age and energy intake. P value less than 0.05 was considered as statistically significant. Q, quartile; SD, stander deviation. 
El Bilbeisi AH (2019) Association of healthy eating index with metabolic syndrome and its components among type 2 diabetes patients in Gaza Strip, Palestine: A cross sectional study

Table 4. Odd ratio and confidence interval for metabolic syndrome and its components across quartiles of HEI-2010

\begin{tabular}{|c|c|c|c|c|c|}
\hline \multicolumn{6}{|c|}{ Quartiles of Healthy Eating Index-2010 } \\
\hline Q1 (Lowest) & Q2 & Q3 & Q4 (Highest) & P value & OR $(95 \% C I)$ \\
\hline \multicolumn{6}{|c|}{ Metabolic syndrome (62.5\%) } \\
\hline 33.0 & 30.9 & 23.8 & 12.3 & 0.001 & $2.260(1.997-2.558)$ \\
\hline \multicolumn{4}{|c|}{ Adjusted* } & 0.001 & $1.804(1.494-2.178)$ \\
\hline \multicolumn{6}{|c|}{ Central obesity (waist circumference $>90 \mathrm{~cm}$ for men or $>80 \mathrm{~cm}$ for women) $(82.2 \%)$} \\
\hline 28.4 & 26.8 & 24.8 & 20.0 & 0.001 & $1.915(1.646-2.227)$ \\
\hline \multicolumn{4}{|c|}{ Adjusted* } & 0.009 & $1.351(1.077-1.694)$ \\
\hline \multicolumn{6}{|c|}{ Triglycerides $\geq 150 \mathrm{mg} / \mathrm{dl}$ or specific treatment for this lipid abnormality $(\mathbf{4 2 . 0} \%)$} \\
\hline 37.3 & 35.1 & 26.8 & 0.8 & 0.001 & $2.517(2.218-2.855)$ \\
\hline \multicolumn{4}{|c|}{ Adjusted* } & 0.001 & $2.270(1.911-2.697)$ \\
\hline \multicolumn{6}{|c|}{ HDL-c $<40 \mathrm{mg} / \mathrm{dl}$ in males and $<50 \mathrm{mg} / \mathrm{dl}$ in females or specific treatment for this lipid abnormality $(40.6 \%)$} \\
\hline 36.3 & 35.9 & 27.4 & 0.4 & 0.001 & $2.412(2.129-2.733)$ \\
\hline \multicolumn{4}{|c|}{ Adjusted* } & 0.001 & $2.089(1.763-2.475)$ \\
\hline \multicolumn{6}{|c|}{ High blood pressure $(\geq 130 / 85 \mathrm{mmHg})$ or treatment of previously diagnosed hypertension $(64.4 \%)$} \\
\hline 30.7 & 31.1 & 24.3 & 13.9 & 0.001 & $1.997(1.771-2.251)$ \\
\hline \multicolumn{4}{|c|}{ Adjusted* } & 0.001 & $1.361(1.145-1.618)$ \\
\hline \multicolumn{6}{|c|}{ In our study, all participants previously diagnosed type 2 diabetes mellitus } \\
\hline
\end{tabular}

The OR and CI for the metabolic syndrome and its components across quartiles of HEI-2010 were tested by binary logistic regression. *Adjusted for age, gender, educational level, monthly income, diabetes duration, type of diabetes medications used, received diabetes care instructions, physical activity level (total MET), body mass index and fasting plasma glucose. P value less than 0.05 was considered as statistically significant. Q, quartile; OR, odds ratio; CI, confidence interval; HDL-c, high-density lipoprotein cholesterol.

\section{Discussion}

This cross sectional study was conducted among 1187 previously diagnosed T2DM (Both genders, aged 20-64 years), patients receiving care in PHCs in Gaza Strip, Palestine. To the best of our knowledge, this is the first study, which describes the association of HEI-2010 score with MetS and its components among T2DM patients in Gaza Strip, Palestine. The main findings of this study indicate that, after adjustment for potential confounders, patients in the lowest quartile of HEI-2010 had the highest risk of (MetS, central obesity, high TGs, low HDL-c and high BP), compared with those in the upper quartile. In fact, very few studies have explored the relationship between HEI-2010 score with MetS and its components in patients with T2DM, which made the comparison of our results with previous studies difficult. Previous studies examining diet have focused on single nutrients, such as proteins [7], carbohydrates [8] and/or dietary fats [9]. However, single nutrients do not measure overall diet quality as well as an examination of the overall diet by HEI $[14,37]$. It is well established that studying whole diet rather than individual foods or nutrients can exhibit overall diet quality better and help predict the association of diet quality with health risks [6]. Diet quality indices are widely used to evaluate the relationship between total diet and chronic diseases risk factors [13]. The HEI-2010 is an updated tool for assessing diet quality as specified by the Dietary Guidelines for Americans, and therefore the high HEI2010 scores are thought to reflect healthier diets in a population [15]. Several prospective studies have evaluated HEI-2010 scores in relation to T2DM [17,38]. Results from a recent meta-analysis of 15 cohort studies, with follow-up time ranging from 5 to $\geq 24$ years, show that diets of the highest quality (Compared highest vs. lowest quintile scores), as assessed by the HEI-2010, are associated with a significant risk reduction for all-cause mortality, T2DM and other chronic diseases [23]. The meta-analysis included seven reported studies on T2DM as the main disease outcome, with age ranging from 30 to 79 years, among individuals from different ethnic groups including Caucasian (European), non-Hispanic White, African American, Hispanic, and Asian; of these studies, the main result indicates that diets that score highly on the HEI-2010 are associated with a significant reduction in the risk of T2DM [23].
In addition, Saraf-Bank et al. [28] in a cross sectional study show that, adherence to HEI-2010 is inversely associated with MetS and its components (Abdominal obesity, high BP, high TGs and low HDL-c) among Iranian adult women. Another study showed that, based on HEI2010 values, low diet quality can be related to MetS and its components [11]. Our results are in line with previous studies that showed a negative association between HEI-2010 and risk of MetS and its components. Furthermore, Tande et al. [39] in a cross sectional study show that, dietary consumption that follows the HEI is associated with a lower risk for abdominal obesity. Cespedes et al. [40] show that, according to HEI2010 values, a three years improvements in diet quality was modestly protective against gain in WC and partially explained by less weight gain among post-menopausal women. Moreover, Schwingshackl et al. [23] indicated that, dietary consumption that follows the HEI is associated with a lower risk for abdominal obesity. Additionally, many of the previous study indicated that dietary consumption that follows the HEI is associated with a lower risk for high TGs level, low HDL-c and high BP $[41,42]$. The results of our study support these findings. On the other hand, the results of our study revealed that, $62.5 \%$ of T2DM patients had MetS based on the IDF criteria. There are several studies reporting prevalence of the MetS in this T2DM population more than double the prevalence in the general populations $[4,5]$. In similar studies amongst T2DM subjects in Nigeria, a high prevalence rate of $62.5 \%$, was seen, while a lower prevalence rate of $56 \%$ was seen in patients of Saudi Arabian origin $[43,44]$. In our study, the high prevalence of MetS could be due to the low level of HDL-c, high BMI and large WC amongst our people, which considered the main risk factors for the MetS.

Additionally, our findings revealed that, patients in the upper quartile of HEI-2010 had higher intakes of carbohydrate, dietary fiber, fruits, vegetables, meat and fish, whole grains, nuts and legumes, compared with those in the lowest quartile. Furthermore, the energy intake, fat and refined grain consumption decreased significantly across HEI-2010 quartiles. Based on the Dietary Guideline for Americans the high HEI-2010 scores are accompanied with high contents of fruits, vegetables and low amounts of saturated fatty acids. The beneficial effects of fruits and vegetables intakes in the framework of dietary patterns were reported previously, and it has been shown that dietary patterns rich in fruit and vegetables may reduce the risk of the MetS 
and its components [6] and are associated with lower concentrations of inflammatory markers [3]. Esmaillzadeh et al [45] show that, higher intakes of fruit and vegetables are associated with a lower risk of the MetS; and the author concluded that, the lower risk may be the result of lower C-Reactive Protein concentrations. In a previous study, we have demonstrated the inverse associations between the Asianlike pattern characterized by a high intake of whole grains, potatoes, beans, legumes, vegetables, tomatoes and fruits with (MetS, central obesity, high TGs, low HDL-c and high BP) [6]. Moreover, another study showed that, higher intakes of hydrogenated vegetable oils were associated with a greater risk of the MetS and insulin resistance, whereas higher intakes of non-hydrogenated vegetable oils, in the range of energy requirements, were associated with a lower risk of insulin resistance [46]. It is assumed that this association could be attributed to healthy ingredients including vitamins, phytochemicals, dietary fibers, potassium, magnesium and antioxidants of fruits and vegetables. These nutrients have been independently associated with reduced metabolic risks related to MetS. Soluble dietary fibers in these foods may decrease the intestinal absorption of cholesterol and bile salts, which may improve serum lipid profile [47]. Magnesium and potassium in many fruits and vegetables could contribute to lower BP [48]. Furthermore, antioxidants in these foods may have beneficial effects on the risk of MetS [49]. Additionally, vegetables and fruits increase satiety and may be associated with a lower risk of obesity [50]. Actually, the underlying cause of desirable association between HEI-2010 with MetS and its components need more studies in the future.

The main limitations of this study is its cross sectional design; the causal relationship could not be determined and it limits the generalizability of our results. In addition, it is possible that unknown or unmeasured confounding variables affect our results. Unfortunately, we do not have measures of total cholesterol, low-density lipoprotein cholesterol and glycated hemoglobin as a marker of diabetes control. The main strength of our study was its being the first study, which shows the association of HEI-2010 with MetS and its components among T2DM patients in Gaza Strip, Palestine, and its large representative sample size. Finally, using a semi-quantitative FFQ that indicates habitual intakes of patients and precise assessment of confounders are the strengths of the present study.

\section{Conclusion}

We conclude that, the highest HEI-2010 score may be associated with a lower prevalence of MetS and its components among T2DM patients. Further prospective cohort studies are needed to confirm the results of this study.

\section{Declarations}

\section{Ethics approval and consent to participate}

The study protocol was approved by the Ethics Committee of Tehran University of Medical Sciences (Code: IR.TUMS.REC.1394.58) and by the Palestinian Health Research Council (Helsinki Ethical Committee of Research PHRC/HC/60/15). In addition, written informed consent was also obtained from each participant.

\section{Consent for publication}

Not applicable.

\section{Availability of data and materials}

The datasets used and/or analyzed during the current study are available from the corresponding author on reasonable request.

\section{Competing interests}

The authors declare that they have no competing interests.

\section{Funding}

This research did not receive any specific grant from funding agencies in the public, commercial, or not-for-profit sectors.

\section{Authors' contributions}

AA and AHB participated in the design of the study, data collection, performed the statistical analysis and drafted the manuscript. $\mathrm{KD}$ and AHB supervising the study and participated in draft review. All authors have read and approved the final version of the manuscript and agree with the order of presentation of the authors.

\section{Acknowledgments}

The authors thank the staff and participants in the Palestinian Ministry of Health, PHCs for their important contributions to the study.

\section{References}

1. Farag HA, Hosseinzadeh-Attar MJ, Muhammad BA, Esmaillzadeh A, El Bilbeisi AH (2019) Effects of vitamin C supplementation with and without endurance physical activity on components of metabolic syndrome: A randomized, double-blind, placebocontrolled clinical trial. Clinical Nutrition Experimental.

2. Suzuki T, Hirata K, Elkind MS, Jin Z, Rundek T, et al. (2008) Metabolic syndrome, endothelial dysfunction, and risk of cardiovascular events: The Northern Manhattan Study (NOMAS). American Heart Journal 156: 405-410.

3. El Bilbeisi AH, Hosseini S, Djafarian K (2017) Association of dietary patterns with diabetes complications among type 2 diabetes patients in Gaza Strip, Palestine: a cross sectional study. Journal of Health, Population and Nutrition 36: 37.

4. Lee WY, Park JS, Noh SY, Rhee EJ, Kim SW, et al. (2004) Prevalence of the metabolic syndrome among 40,698 Korean metropolitan subjects. Diabetes Research and Clinical Practice 65: 143-149.

5. Uzcátegui E, Valery L, Uzcátegui L, Pérez RG, Marquina D, et al. (2015) Prevalence of the metabolic syndrome, insulin resistance index, leptin and thyroid hormone levels in the general population of Mérida (Venezuela). Investigacion Clinica 56: 169-181.

6. El Bilbeisi AH, Hosseini S, Djafarian K (2017) Dietary patterns and metabolic syndrome among type 2 diabetes patients in Gaza Strip, Palestine. Ethiopian Journal of Health Sciences 27: 227-238.

7. Wang ET, de Koning L, Kanaya AM (2010) Higher protein intake is associated with diabetes risk in South Asian Indians: The metabolic syndrome and atherosclerosis in South Asians living in America (MASALA) study. Journal of the American College of Nutrition 29: 130-135.

8. Gadgil MD, Anderson CA, Kandula NR, Kanaya AM (2014) Dietary patterns in Asian Indians in the United States: an analysis of the metabolic syndrome and atherosclerosis in South Asians Living in America study. Journal of the Academy of Nutrition and Dietetics 114: 238-243.

9. Poudyal H, Brown L (2015) Should the pharmacological actions of dietary fatty acids in cardiometabolic disorders be classified based on biological or chemical function? Progress in Lipid Research 59: 172-200.

10. El Bilbeisi AH, Albelbeisi A, Hosseini S, Djafarian K (2019) Dietary pattern and their association with level of asthma control among patients with asthma at al-shifa medical complex in Gaza Strip, Palestine. Nutrition and Metabolic Insights 1178638819841394

11. Yosaee S, Erfani M, Bazrafshan MR, Entezami N, Alinavaz M, et al. (2017) Correlation between diet quality and metabolic syndrome. Journal of Nutrition and Food Security 2: $213-220$

12. Kastorini CM, Milionis HJ, Esposito K, Giugliano D, Goudevenos JA, et al. (2011) The effect of Mediterranean diet on metabolic syndrome and its components: a metaanalysis of 50 studies and 534,906 individuals. Journal of the American College of Cardiology 57: 1299-1313.

13. Khakpouri S, Safari M, Ghazizadeh H, Parizadeh SM, Nematy M, et al. (2019) The relationship between the healthy eating index and an alternate healthy eating index with the risk factors for cardiovascular disease in a population from northeastern Iran. Translational Metabolic Syndrome Research 2: 1-6. 
El Bilbeisi AH (2019) Association of healthy eating index with metabolic syndrome and its components among type 2 diabetes patients in Gaza Strip, Palestine: A cross sectional study

14. Guenther PM, Kirkpatrick SI, Reedy J, Krebs-Smith SM, Buckman DW, et al. (2013) The Healthy Eating Index-2010 is a valid and reliable measure of diet quality according to the 2010 Dietary Guidelines for Americans. The Journal of Nutrition 144: 399-407.

15. Guenther PM, Casavale KO, Reedy J, Kirkpatrick SI, Hiza HA, et al. (2013) Update of the healthy eating index: HEI-2010. Journal of the Academy of Nutrition and Dietetics 113: 569-580.

16. Hiza HA, Casavale KO, Guenther PM, Davis CA (2013) Diet quality of Americans differs by age, sex, race/ethnicity, income, and education level. Journal of the Academy of Nutrition and Dietetics 113: 297-306.

17. Chiuve SE, Fung TT, Rimm EB, Hu FB, McCullough ML, et al. (2012) Alternative dietary indices both strongly predict risk of chronic disease. The Journal of Nutrition 142: 1009-1018.

18. Reedy J, Krebs-Smith SM, Bosire C (2010) Evaluating the food environment: application of the Healthy Eating Index-2005. American Journal of Preventive Medicine 38: 465-471.

19. Glanz K, Hersey J, Cates S, Muth M, Creel D, et al. (2012) Effect of a nutrient rich foods consumer education program: results from the nutrition advice study. Journal of the Academy of Nutrition and Dietetics 112: 56-63.

20. Rehm CD, Monsivais P, Drewnowski A (2011) The quality and monetary value of diets consumed by adults in the United States. The American Journal of Clinical Nutrition 94: 1333-1339.

21. Seymour JD, Calle EE, Flagg EW, Coates RJ, Ford ES, et al. (2003) Diet quality index as a predictor of short-term mortality in the American Cancer Society Cancer Prevention Study II Nutrition Cohort. American Journal of Epidemiology 157: 980-988.

22. Guenther PM, Reedy J, Krebs-Smith SM (2008) Development of the healthy eating index-2005. Journal of the American Dietetic Association 108: 1896-1901.

23. Schwingshackl L, Hoffmann G (2015) Diet quality as assessed by the healthy eating index, the alternate healthy eating index, the dietary approaches to stop hypertension score, and health outcomes: a systematic review and meta-analysis of cohort studies. Journal of the Academy of Nutrition and Dietetics 115: 780-800.

24. Onvani S, Haghighatdoost F, Surkan PJ, Larijani B, Azadbakht L (2017) Adherence to the Healthy Eating Index and Alternative Healthy Eating Index dietary patterns and mortality from all causes, cardiovascular disease and cancer: a meta-analysis of observational studies. Journal of Human Nutrition and Dietetics 30: 216-226.

25. El Bilbeisi AH, Shab-Bidar S, Jackson D, Djafarian K (2017) The prevalence of metabolic syndrome and its related factors among adults in Palestine: a meta-analysis. Ethiopian Journal of Health Sciences 27: 77-84.

26. Naja F, Hwalla N, Itani L, Baalbaki S, Sibai A, et al. (2015) A novel Mediterranean diet index from Lebanon: comparison with Europe. European Journal of Nutrition 54: 1229-1243.

27. El Bilbeisi AH, Srour M, el Afifi A, Farag HA, Djafarian K (2019) Dietary patterns and their association with depression among type 2 diabetes patients in Gaza Strip, Palestine. Food and Nutrition 10: 533-550.

28. Saraf-Bank S, Haghighatdoost F, Esmaillzadeh A, Larijani B, Azadbakht L (2017) Adherence to Healthy Eating Index-2010 is inversely associated with metabolic syndrome and its features among Iranian adult women. European Journal of Clinical Nutrition 71: 425.

29. Pennington JA (2002) Food composition databases for bioactive food components. Journal of Food Composition and Analysis 15: 419-434.

30. Djafarian K, Hosseini S, el Bilbeisi AH (2017) The prevalence of malnutrition and associated factors among hemodialysis patients at al-shifa medical complex in Gaza Strip, Palestine. International Journal of Hospital Research 6: 36-44.

31. El Bilbeisi AH, Hosseini S, Djafarian K (2018) Prevalence of metabolic syndrome and its components using two proposed criteria among patients with type 2 diabetes in Gaza Strip, Palestine. BAOJ Nutrition 4: 054.
32. Farag HA, Hosseinzadeh-Attar MJ, Muhammad BA, Esmaillzadeh A, El Bilbeisi AH (2018) Comparative effects of vitamin D and vitamin C supplementations with and without endurance physical activity on metabolic syndrome patients: a randomized controlled trial. Diabetology \& Metabolic Syndrome 10: 80.

33. El Bilbeisi AH, Hosseini S, Djafarian K (2018) Dietary patterns and their association with blood pressure control among hypertensive patients in Gaza Strip, Palestine. Journal of Family Medicine and Health Care 4: 5-12.

34. El Bilbeisi AH, Hosseini S, Djafarian K (2017) The association between physica activity and the metabolic syndrome among type 2 diabetes patients in Gaza strip, Palestine. Ethiopian Journal of Health Sciences 27: 273-282.

35. Farag HA, Hosseinzadeh-Attar MJ, Muhammad BA, Esmaillzadeh A, el Bilbeisi AH (2019) Effects of vitamin D supplementation along with endurance physical activity on lipid profile in metabolic syndrome patients: A randomized controlled trial. Diabetes \& Metabolic Syndrome: Clinical Research \& Reviews 13: 1093-1098.

36. Khodarahmi M, Asghari-Jafarabadi M, Abbasalizad Farhangi M (2019) A structura equation modeling approach for the association of a healthy eating index with metabolic syndrome and cardio-metabolic risk factors among obese individuals. PloS One 14:e219193.

37. Kant AK (2004) Dietary patterns and health outcomes. Journal of the American Dietetic Association 104: 615-635.

38. McCullough ML, Feskanich D, Stampfer MJ, Giovannucci EL, Rimm EB, et al (2002) Diet quality and major chronic disease risk in men and women: moving toward improved dietary guidance. The American Journal of Clinical Nutrition 76: 1261-1271.

39. Tande DL, Magel R, Strand BN (2010) Healthy Eating Index and abdominal obesity. Public Health Nutrition 13: 208-214.

40. Cespedes Feliciano EM, Tinker L, Manson JE, Allison M, Rohan T et al. (2016) Change in dietary patterns and change in waist circumference and DXA trunk fat among postmenopausal women. Obesity 24: 2176-2184.

41. Nicklas TA, O'Neil CE, Fulgoni III VL (2012) Diet quality is inversely related to cardiovascular risk factors in adults. The Journal of Nutrition 142: 2112-2118.

42. Haghighatdoost F, Sarrafzadegan N, Mohammadifard N, Sajjadi F, Maghroon M, et al. (2013) Healthy eating index and cardiovascular risk factors among Iranians. Journal of the American College of Nutrition 32: 111-121.

43. Unadike BC, Akpan NA, Peters EJ, Essien IO, Essien OE (2009) Prevalence of the metabolic syndrome among patients with type 2 diabetes mellitus in Uyo, Nigeria. African Journal of Endocrinology and Metabolism 8: 9-11.

44. Akbar D (2002) Metabolic syndrome is common in Saudi type 2 diabetic patients. Hypertension 12: 47-49.

45. Esmaillzadeh A, Kimiagar M, Mehrabi Y, Azadbakht L, Hu FB, et al. (2006) Frui and vegetable intakes, C-reactive protein, and the metabolic syndrome. The American Journal of Clinical Nutrition 84: 1489-1497.

46. Esmaillzadeh A, Azadbakht L (2008) Consumption of hydrogenated versus nonhydrogenated vegetable oils and risk of insulin resistance and the metabolic syndrome among Iranian adult women. Diabetes Care 31: 223-226.

47. Visioli F (2011) Nutritional support in the pharmacological treatment of metabolic syndrome. European Journal of Pharmacology 668:S43-9.

48. Savica V, Bellinghieri G, Kopple JD (2010) The effect of nutrition on blood pressure. Annual Review of Nutrition 30: 365-401.

49. Czernichow S, Vergnaud AC, Galan P, Arnaud J, Favier A, et al. (2009) Effects of longterm antioxidant supplementation and association of serum antioxidant concentration with risk of metabolic syndrome in adults. The American Journal of Clinical Nutrition 90: 329-335.

50. Deshmukh-Taskar PR, O’Neil CE, Nicklas TA, Yang SJ, Liu Y, et al. (2009) Dietary patterns associated with metabolic syndrome, sociodemographic and lifestyle factors in young adults: the Bogalusa heart study. Public Health Nutrition 12: 2493-2503.

Copyright: (C2019 El Bilbeisi AH. This is an open-access article distributed under the terms of the Creative Commons Attribution License, which permits unrestricted use, distribution, and reproduction in any medium, provided the original author and source are credited. 